\title{
Téoros
}

Revue de recherche en tourisme

\section{La recherche documentaire en tourisme : les outils informatisés}

\section{Hélène Bussière}

Volume 14, numéro 3, automne 1995

Tourisme et technologies de l'information

URI : https://id.erudit.org/iderudit/1075084ar

DOI : https://doi.org/10.7202/1075084ar

Aller au sommaire du numéro

Éditeur(s)

Université du Québec à Montréal

ISSN

0712-8657 (imprimé)

1923-2705 (numérique)

Découvrir la revue

Citer cet article

Bussière, H. (1995). La recherche documentaire en tourisme : les outils informatisés. Téoros, 14(3), 26-30. https://doi.org/10.7202/1075084ar d'utilisation que vous pouvez consulter en ligne.

https://apropos.erudit.org/fr/usagers/politique-dutilisation/ 


\section{LA RECHERCHE}

\section{DOCUMENTAIRE

\author{
Hélène Bussière \\ est bibliothécaire de référence, \\ Service des bibliothèques, \\ Université du Québec à \\ Montréal (e.mail: \\ bussiere.helene.uqam.ca)) \\ (http: www.uqam.ca)
}

Dans ce présent article, nous almerlons tracer un portralt de la sttuation quI prévaut dans les blbllothèques, lesquelles, nous le verrons. n'ont d'autres cholx que d'Intégrer les nouvelles technologles de l'Information. A l'heure de la numérlsation de l'Information et de l'Inforoute, quelles sont les tendances concernant la documentation qui depuls toujours repose sur l'Imprimé? Quelle est la place occupée par l'Imprimé par rapport à celle qu'occupent présentement les outlls Informatıses dans les bibllothèques unlversitalires?

Par alleurs, nous examinerons les avantages et les Inconvénlents que présentent, pour le chercheur, les outlls de recherche imprimés et informatisés. Nous utilisemons pour ce falre les critères sulvants: les mises à jour, le mode de recherche, les Interfaces et enfln les caractéristiques addlltionnelles. Enfln, nous désirons offrir au lecteur un panorama des outlls de recherche Informatısés appllqués au domalne du tourisme: quelles sont les ressources disponlbles et les contenus offerts par celles-cl. Et parml la gamme de supports offerts, nous nous demanderons ce que préfèrent les princlpaux Intéressés: les utllisateurs. Mals avant tout, qu'en est-1l de la situation dans les blbllothèques unlversltalres face aux nouvelles technologles de l'information?

\section{Les outils de recherche imprimés vs les outils informatisés: plus qu'une rivalité commerciale?}

Face aux nouvelles technologles et plus particullèrement aux réseaux électronlques d"Information, plusleurs prévolent une métamorphose radicale des blbllothèques: seront-elles dans un proche aventr sans papler? Selon une étude réallsée par Apple, II y avalt en 1994 plus de 5 millions de lecteurs de disques compacts (CD-ROM) dans le monde, avec des perspectlves évaluées à 15 millions pour 1995. C'est dire à quel polnt les disques compacts ont falt une percée dans tous les domalnes: le monde des affalres, celul des professionnels, les milleux éducatifs et aussi les foyers. Le multimédla qui combine le son, des images animées ou non et de l'informatuon textuelle par le blals d'un environnement informatique a envahl tous les domalnes de la connalssance en ralson justement de son "mervelleux emballage pour l'Information"${ }^{1}$ (Yager, 1992). Les avantages que possède cette technologle et sa diffusion de plus en plus répandue font de ces ressources un sérleux rlval à l'Imprimé. Le dlctlonnalre Webster par exemple, maintenant offert sur CD-ROM, permet d'entendre la prononclation d'un mot. ce que ne peut offrir la verslon.Imprimée. Ron Palmich-2 (PalmIch, 1991) qul a

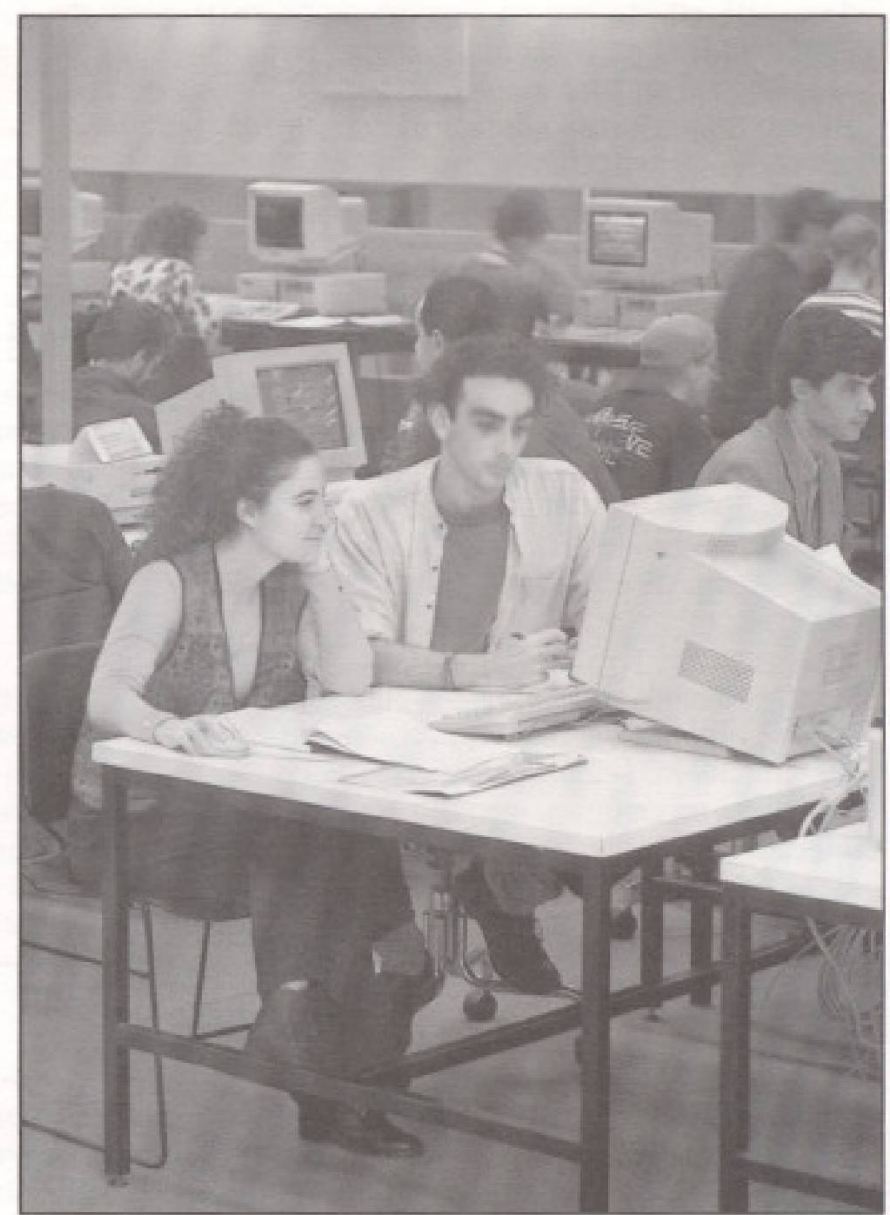

Photo: Information externe, UQAM

mené une étude sur l'interactivité a peut-être une expllcation pour cet engotement. En effet, l'être humain possède des capacités de rétension limitées: Il ne retlent que $25 \%$ de ce qu'll volt, $40 \%$ de ce qu'll volt et entend, et $75 \%$ de ce qu'll volt, entend et falt. Louls Le Borgne- ${ }^{3}$ (Le Borgne, 1993) trace alnsl les limites de l'imprimé par rapport aux outils polymorphes (son, Image fixe ou animée, couleurs, symboles, etc): "On dolt donc admettre que la transmission et la converslon de cette masse d'Information sur papler sont non seulement devenues des défls carrément Insurmontables avec les moyens tradituonnels, mals aussl que cette informatlon devient de molns en molns repérable ou Identiflable dans des conditlons temporellement et économiquement utiles." En effet. les réseaux Informatliques déflent les lim ites du temps quant à la transmission de documents car ceux-cl peuvent être transmIs, presque instantanément, d'un bout à Tautre de la planète. Jean-Plerre Côté, directeur général du Service des BIbllothèques à l'UQAM et Rélean Bernard. 
directeur du Service des télécommunicatlons à la même Instıtution, s'ont appelés à se prononcer régullèrement sur les réseaux informatiques et le rôle que ces dernlers sont appelés à fouer: "C'est blen plus qu'une questlon technologique. On parle plutôt de transformations profondes de nature soclale et culturelle. La survle mểme de la démocraLle est, en jeu."-4 (Ravary, 1994). Ne rlsque-t-on pas en effet de créer une soclété à deux pallers: les "Info-riches" et les "Info-pauvres"? D'où la nécessité pour les blbllothèques. seul endrolt à offrlr des servilces gratuilts, de prendre le virage de I'Inforoute. C'est d'allleurs dans ce contexte qu'est évoquée la notıon de "blbllothèque virtuelle". En réalité, nous sommes délà montés dans le train de l'inforoute. En effet. II est maintenant posslble, grâce aux nouvelles teclmologles de l'Information, de consulter alsément et rapidement des catalogues de bibllothèques et de centres de recherche et ce, à travers le monde. De plus, par le blals des technlques de numérisation, les documents eux-mêmes, locallsés à l'échelle planétalre, sont à notre portée vla les réseaux informatiques. Les chercheurs ont la possiblité de communlquer le frult de leurs recherches par courrter électronlque et non plus seulement par l'Intermédlalre de revues savantes. Enfln les coûts élevés de la documentation obllgent les Inst.tutıons à repenser leurs modes de transm Ission de l'information et à uthlser de nouveaux moyens pour chercher cette documentathon et la mettre à la disposition des usagers.

Nous sommes donc dans une pérlode de mutation qul ne se IImite pas qu'aux seules frontlères des blbllothèques: certes. le monde de l'édition est en profonde transformation mals les nouvelles technologles et les réseaux informatiques bouleversent nos modes traditionnels de communlcation sl blen que l'imprimé n'est plus le seul support propageant le savoir. Mals délalssons pour le moment ce débat pour tenter d'évaluer les avantages et les Inconvénlents de l'imprlmé par rapport aux outils informatisés. Posons d'abord la questlon de l'actualité des ressources ou de la fréquence des mlses à jour de l'Informatlon dans les outils de recherche.

\section{Les mises à jour}

Avant la percée spectaculaire du CD-ROM en blbllothèque. les professionnels de l"Information Interrogenlent, à distance pour les chercheurs des bases de domées en llgne par le blals des systèmes de télécommunlcatlon. Ce type d"interrogation appelé "téléréférence" existe toujours. Cependant depuls l'implantation de services de CD-ROMs dans les blbllothèques, on y falt de molns en moins appel. En effet, ces bases de données, quil sont en grande ma|orité des index d'articles de pérlodlques, sont commerclalisées sur CD-ROM. Pour les blbllothèques, Il exlste de nombreux avantages à utiliser les CD-ROMs par rapport à la téléréférence. Le plus Important est sans doute le princlpe de tarification des CDROMs qul est flxe (abonnements annuels). La tariflcatlon de la téléréférence se calcule quant à elle au temps d'interrogatlon, au nombre de références obtenues et au nombre de références télédéchargées ou sauvegardées. Avec la téléréférence, une simple recherche peut facllement s'élever à plus de cent dollars. Tandls que le cott d'interrogatlon des CD-ROMs est transparent pour le chercheur.

Les mIses à jour des CD-ROMs sont toutefols beaucoup molns fréquentes que les bases de données en ligne: la plu- part sont trimestrlelles et quelques-unes annuelles. En ligne, les mises à jour sont en majorlté mensuelles, quelques-unes sont hebdomadaires et d'autres quot.ldiennes. C'est Icl que nous pouvons parler de complémentarité des différents supports. Un chercheur soucleux d'obtentr les informations les plus à four possibles pourra Interroger sur CD-ROM et faire effectuer une recherche en ligne pour obtenlr les références les plus récentes. SI nous examinons des bases de données de façon plus précise, par exemple REPÈRE permettant de repérer par sujet des références d'articles de pérlodiques francophones, la plus récente mlse à four de ce CD-ROM couvre la pérlode allant de 1980 à 1994 . Lactuallté de ce CD-ROM et la pérlode rétrospective qu'll couvre rivallsent difficllement avec son équivalent Imprimé Polnt de Repère dont Il est possible de consulter le mols de Juin 1995 alors que nous sommes en août. landls que sa pérlode rétrospective va blen en deca de 1980 . Toutefols, II existe des bases de données sur CD-ROM qul n'ont pas d'équlvalents, nl en ligne, nl en Imprimé. C'est le cas d'URBADISC couvrant la pérlode de 1970 à 1994 . Soullgnons que les tendances du marché font que les Index n'ayant pas d'équivalents sur GD-ROM sont de plus en plus rares.

Par allleurs, on peut remarquer que les mlses à jour des CDROMs ont tendance à s'améllorer au fur et à mesure que les coûts de production dimimuent et quaugmentent les demandes des utllisateurs. En falt, rien n'est très simple en ce qui concerne les mises à four... et le monde de l'information en général! En effet. nous avons vu que la complémentarité des dlfférents supports est un aspect important à consldérer et à évaluer pour chaque recherche. Les blbllothécalres sont formés pour gulder et conseller les chercheurs dans toutes leurs démarches documentalres. Ils sont donc les melleurs guldes quant aux mlses à jour fles différents supports disponibles.

S'il existe encore des lacunes quant aux mises à jour et aux pérlodes rétrospectives des bases de données, ces dernlères comportent de nombreux avantages notamment celul du mode de repérage que nous nous proposons d'examlner malntenant.

\section{Le mode de repérage}

Les out.lls informatisés (en llene ou sur CD-ROM) possèdent de grandes qualités quant au mode de repérage: Ils sont plus rapldes et lls offrent des accès de recherche qul ne se limltent pas qu'au sujet. Précisons tout d'abord que toutes les bases de données sont structurées en champs dont les plus communs sont: l'auteur, le titre, le titre du pérlodlque, le sujet décrit par des mots-clés, l'année de publication. Tous ces champs sont Intermgeables, c'est-à-dire qu'll est posslble d'interroger par le nom d"un auteur, par mot-clé, par titre de pérlodlque, par année de publlcatlon. etc. Il est aussl possible de comblner plusleurs champs de recherche. Les Index Imprimés sont loin de posséder une telle flexibilité pulsque les références sont repérables que sous un mot-clé rangé dans l'ordre alphabétique.

Toujours dans le contexte des outlls Informatısés, les opérateurs booléens (ET, OU, SAUF) permettent. à l'utIlisateur de comblner des mots-clés. Par exemple: "marketing et tourisme" ou encore. "site ou espace" et "aménagennent". Un 
seul ennul mals de talle, les ut.lisateurs novices confondent souvent le "et" et le "ou" loglque-5 (Cooper. 1988) ce quil blen sûr pose problème quant aux résultats obtenus lors des Interrogations. Notons que de plus en plus de loglciels d'Interrogation permettent l'utilisation du langage naturel ce qui permet de contourner la difflculté d'utulısatlon des opérateurs booléens? En outre, certalnes bases de données offrent la possibilité au chercheur de spécifler l'adjacence des mots permettant alnsl de repérer des références contenant deux mots sltués à proxlmité l'un de l'autre.

La troncature de fin de mot ajoute une autre souplesse aux prodults Informatisés: "hôtel?" par exemple permettra à l"utllisateur de retrouver des références où les mots hôtel. hôtels, hôteller, hôtellı̀re et hôtellerle apparaîtront. La troncature à l'intérleur des mots peut aussl être disponlble selon les prodults. Encore une fols, de telles flexiblittés n'exIstent pas avec les Index Imprimés.

Comparés aux outlls Imprimés, le potentlel de recherche offert par les outIIs Informatisés est Impressıonnant lorsqu'lls sont utulisés adéquatement. Un chercheur Initlé à I'Interrogation des CD-ROMs peut difficllement revenlr aux Index Imprimés à molns d'y être contralnt faute d'équlvalent sur CD-ROM. Mals tel que mentıonné plus haut. cette sluantlon est de plus en plus rare.

\section{Les interfaces}

Rappelons que ce sont les utIllsateurs qui, avec beaucoup de satisfaction, effectuent eux-mêmes leurs recherches avec les CD-ROMs. Il existe pour le moment un problème de normallsation quant aux interfaces appelés aussi logiclels de navlgatlon. Cette diversité des logiclels obllgent les utIlisateurs à apprendre plusleurs types de commandes. Face à cette diversité, le danger qui guette l'utullsateur sporadlque est son perpétuel apprentlssage. Avec ralsons, les blbllothécalres se plalgnent de cette situation. Ils dolvent donc multIpller les outlls d'alde: guldes d'utllisation, formatlons. présence constante d'employés dans les servlces de CDROMs. Soullgnons que tous les outlls Informatlsés possèdent, à l'écran, une alde contextuelle. Cependant l'expérlence que nous avons acqulse au cours des derulères années au service des CD-ROMs de I'UOAM, nous permet d'afTrmer que l'alde humalne est encore celle qul est le plus souvent soulaltée et sollicltée. Un autre constat: toutes les subtulltés que peuvent présentées les outlls informatısés et énumérées plus haut sont souvent Ignorés par le chercheur novice. Nous ne pouvons d'allleurs qu'encourager une plus grande particlpation des utillsateurs aux formatlons documentalres. Dans le même sens, une volonté. au nlveau gouvernemental. d"Intégrer la formatlon documentalne à la ve scolalre et ce, dès le primaire, ne serait que soulıaltable.

\section{Caractéristiques additionnelles}

Un autre avantage Indénlable des outils Informatisés par rapport aux outlls Imprimés est qu'lls offrent la possibilité d'lmprimer ou de sauvegarder sur disquettes les références obtenues à l'écran. Cette dernlère posslbilıté permet de récupérer le ou les flchlers avec un traltement de textes ou encore par le blals de logiclels conçus pour gérer des blbllographles tel que Proclte.
Les avantages que comportent les outlls sur CD-ROMs et la satisfactlon des utllisateurs pour ceux-cl ont rapidement rendu marglnaux les supports concurrents: papler. microphorme, ordinollngue. Les applications en blbllothèque đes CD-ROMs s'étant révélées un succès, on retrouve malntenant toute une gamme de prodults sur ce support: Index d'articles de pérlodiques, des répertoires de thèses ou de llvres disponibles, des répertolres de statistique ou encore de données numérlques, de cartes géographlques, des dlctlonnalres, des encyclopédles, etc. De plus, Il est aussı posslble de retracer non seulement des références à des articles de revues ou de journaux, mals aussi des articles en version Intégrale ou en pleln texte.

Comprenons qu'll ne s'aglt pas dans cet article de vendre la cause des CD-ROMs. Elle est délà vendue et blen Implantée dans les blbllothèques unlversitalres. C'est d'allleurs ce que démontre les études menées sur les préférences des utllisateurs par rapport à différents supports. Parce qu'ils permettent de repérer I'lnformatlon beaucoup plus rapldement et plus efflcacement, les utllisateurs préfèrent les ressources Informatısées. Ils préfèrent aussı les CD-ROMs aux systèmes en ligne, en ralson de leur gratulté. A l'université Columbla- ${ }^{6}$ (Juhl, Lowry. 1990). $71 \%$ des usagers préferent les CDROMs aux outlls Imprimés. Une autre étude menée cette fols à Oakland-7 (Schultz, Salomon, 1990) conclut que les utilisateurs étalent très satlsfalts des CD-ROMs pour les ralsons sulvantes: $83 \%$ d'entre eux estlmalent économlser du temps, $60 \%$ se dlsalent satusfalent des résultats de leur recherche. et $85 \%$ les préféreralent aux Index Imprimés. Citons une dernlère étude réallsée à l'unlversité Illinols- ${ }^{-8}$ (Allen, 1989) où les étudlants ont tous sans exception préféré les CDROMs aux Index Imprimés. Examinons maintenant quelles sont les ressources informatlsées que l"on peut consulter en tourlsme.

\section{Les outils informatisés en tourisme}

Liédition 1995 The CD-ROM Directory-5 contenalt plus de 8,000 entrées et descriptions de CD-ROMs et d'outils mutımédla. Pour qul veut une vue d'ensemble des prodults offerts sur le marché, ce répertolre se révèle un outll Incontournable. Soullgnons que d'autres répertolres de même type existent-10. The CD-ROM Directory présente un Index sujet dont celui du tourisme: on y retrouve plus de 154 tutres. Ces titres sont tantôt des guldes touristlques (Hong Kong at ils best, Le Guide des Musées de France, National Parks = The Multimedia Family Guide, etc) des atlas, des guldes divers ou spéclalisés pour une cllentèle partlcullère (International Herald Tribune Guide to Business Travel In Europe) des répertolres (Hotel CD-ROM) et des Index d'articles de pérlodiques (The Hopitality Index). II exlste toutefols plusleurs autres CD-ROMs couvrant d'autres disclpllnes ou sujets que le tourlsme quil peuvent être judlcleusement consultés par les chercheurs. Pour des fins de clarté, nous énumèrerons différentes bases de données par catégorle.

\section{Les bases de données bibllographiques}

Dans cette premlère catégorle, les Index dartıcles de pérlodlques occupent une place majorltaire. Ils permettent de repérer par sujet ou par d'autres cliamps tel que mentlonné plus lauıt, des références à des artıcles de pérlodlques ou de 
Journaux. Mals ces Index sont de plus en plus hybrides: II arrive qu'll solt possible de repérer d'autres types de dociments tels que des rapports, des thèses, des llvres, etc. Par allleurs, les frontlères entre les discipllnes étant de inolns en molns étanches, plusleurs prodults peuvent répondre de diverses façons aux besolns des chercheurs en tour'sme. En volcl quelques exemples.

ABI-INFORM: ce CD-ROM dépoulle plus de 800 pérlodlques spéclallsés en gestlon. Les aspects management des services tourlstlques y est très blen couvert. II dépoullle des pérlodlques aussı varlés que Fortune, Food Marketiıg, Hotel \& Motel Management, Canadian Business Review, etc).

Canadian Business and Current Affairs (CBCA): dépoullle des revues et des journaux canadlens anglals. C'est donc un Index général: le quotıdien Globe \& Mall et la revue Maclean's par exemple sont des documents dont les références sont dlsponlbles sur ce CD-ROM.

FRANCIS: prodult en France, ce CD-ROM couvre le domaine des sclences soclales et humalnes dans le contexte européen. Il possède la partıcularité de présenter des références non seulement à des artlcles de pérlodlques mals encore, des rapports, des textes de conférences ou des thèses européennes.

The Hospitality Index: ce CD-ROM couvre plus spéclflquement le domalne de l'industrle hospltallère et tour'stique. II dépoullle plus de 50 pérlodlques dans ce secteur: Tourism management, Journal of Leisure Research, Jourmal of Travel and Tourism Marketing, etc.). On y retrouve auss! des textes de conférence.

PAIS: permet de retracer la documentation commanditée ou produlte par l'adminlstration amérlcalne. Articles de pérlodlques, Ilvres, publlcatlons gouvernementales sont repérables avec ce CD-ROM. Notons que ce prodult a une couverture Internationale.

PSYCLIT: présente des réféfences à des artıcles de pérlodlques et à des llvres dans le domalne de la psychologle et dans des domaines connexes. Pour la psychologle du consommateur ou les aspects marketing par exemple, ce CDROM est tout indiqué.

REPERE: les 480 revues de langue françalse dépoullées par ce CD-ROM couvrent tous les domalnes. Le Nouvel Observateur, Annales de Géographle, L'Actualité, Téoros, Géo, sont quelques-uns des pérlodlques recensés par REPERE. La version actuelle permet en outre de consulter 5,000 verslons intégrales d'artıcles de pérlodiques.

SOCIOFILE: ce CD-ROM est spéclallsé en soclologle et donne accès à des articles de pérlodlques, des llvres, des thèses et des textes de conférences.

SPORT DISCUS: couvre entre autres le domaine des lolsirs et permet de repérer des références à des artıcles de pérlodiques, des llvres, des thèses et des rapports.
URBADISC: est spéclallsé en urbanlsme mals le volet tourlsme occupe une couverture Intéressante. Ce CD-ROM est développé en France sl blen que le contexte européen est abondamment développé. Références à des artıcles de pérlodliues, de llvies et de rapports.

Les bases de données numérlques et/ou statistiques Les chercheurs en tourisme pourront tirer proflt de consultatlons des données statlstlques. II exlste de par le monde de nombreux organlsmes produlsant des statistlques disponlbles de plus en plus sur CD-ROM. Dans la majorité des cas, les domnées sont récupérables à l'alde d'un chıffr'ler tel que le loglclel LOTUS 1-2-3

RECENSEMENT CANADA: équlvalent de l'Imprimé Recensement Canada. Ce CD-ROM comprend des données soclo-économlques et démographıłques de la population canadlenne.

CANSIM: les données produltes par Statistıque Canada peuvent être Interrogées à partir de la base de données CANSIM. Notons que ce CD-ROM contlent des données actuelles et chronologlques couvrant une vaste gamme de sujets soclo-économlques dont le transport, les voyages Internationaux, l'Industrle des services, allments et agriculture, la population pour n'en nommer que quelques-uns.

LA VITRINE DES RÉGIONS: prodult par la Malson des Réglons du Québec, ce CD-ROM présente un vaste portralt de l'ensemble des actlvités soclo-économlques du Québec. Il est possıble de récupérer des données sur le nombre d'habltants dans une munlclpalité donnée, de connaître le nom du malre de celle-cl, le nombre de sites touristlques, de parcs Industrlels, quelles sont les usines qul produlsent du carton par exemple. où sont-elles situées. etc.

\section{Les répertoires}

Le marché des répertolres sur CD-ROM est très varlé: bottin téléphonlque. IIste d'Institutions, liste de membres, de livres, etc. En volcl quelques-uns qul peuvent être utlles pour le chercheur en tourisme.

BOOKS IN PRINT PLUS: contlent tous les livres disponibles en langue anglalse. II contlent de nombreux Index: auteur, titre, sujet en sont les princlpaux. Un chercheur pourra donc recenser tous les llvies sur le marché sur un sujet donné.

DISSERTATION ABSTRACTS INTERNATIONAL: répertorle toutes les thèses amérlcalnes depuls 1861: Index auteur, titre, sujet. La consultation de ce répertolre permet entre autres de vérifler le caractère orıginal d'un sujet.

ELECTRE Biblio: équivalent francophone de Books in Print mentıonné plus haut. II présente tous les livres disponlbles en langue françalse: Index auteur, tutre, sujet.

\section{Les ouvrages de référence}

Ce marché est en plelıe effervescence: dlctlonnalre, encyclopédle, almanach, etc. Les répertolres de CD-ROMs cltés plus haut pourront être consultés par le lecteur afln d'y repérer des titres concernant leurs Intérêts. Mentionnons que le dictlomialre Le Grand Robert est malntenant disponlble sur CD-ROM: Le Robert électronique. 


\section{Les CD-ROMs plein texte}

Les CD-ROMs plésentant des documents of'lgluaux on e.l versıon Intégrale sont de plus en plus présent.s sur le marchoé. Les quotidlens, les revies en Format électronkque sont en effet de plus en plus populaires car on pent relracer non seule inent les références mals aussi le texle de Iarticle -u du document Intégralement. Le chencheur souve IsI un temps précleux putsqu'll ria plus beslon de chercher en bilbllothèque le quolstlen, la nevue oul le document paur le consulter. En effel le chercheur peut les consulter à l'écran. Voyons les CD-ROMs qui présentent un intérêt. primorillal en tourlsine.

BPO: est le titre qul correspond au CD-ROM ABI+NFORM décrlt plus haut. BPO présente envionn 400 titres de revnes en pleln texte. Ce (jul représente la inoltlé des perlordlques dépoulliées par cet Index spéclallsé en gestion.

ACTUALTÉ OUÉBEC: textes et Indlex des pr'Inc]paux quotsdiens québécols: La Presse, Le Devolr, Le Journal de Montréal, Le Solell.

REPERE: tel que ment.ıonné plus ltaut. REPERE contle.ıt environ 5.000 articles en verston intégrale.

\section{Les catalngues de bibliothèques}

Plusteurs inst.tut.lons ont dé|à reconirs au CD-ROM pour présenter leurs catalogues all public. Ces catalogues domnent accès aux collections des Institutlons: Ilvres. alyot!nements aux pérlorllques on aux jonrianx, vildécos, documents de reférence, etc. Mals une autre ressource permet la consultat.Ion des catalonues de blbllothèrues. II s'aglt du réseau INTERNET avec lequel nous almerions conclure ce présent article.

\section{Les perspectives d'aventr dans le domalne de la slocu- mentation}

II est blent diffuclle de flxer l'avenlr dans le domaine de la documentatlon. Les blbliotjè̀ques sont présentement dans un tournant qul semble condanner l'imjpriné an proflt de rien de moins qu'un unvers numértsé. Le réseau INTERNET est à l'lieure actuelle le plus bel exemple d'informations virtuelles. Présentement cette Information semble se développer de façon anarchlıque. Mals an fur et à mesure qu'elle sera plıs structurée et donc plus facilement repérable, ce type d'accès à l'Informatton pirendra sans doute. une tres grande Importance dans le dornalne documentatre.

Le réseau INTERNET est le plus grand réseau de conmunlcation lulormatique qui exlste. On cotuptait environ $29 \mathrm{~m}$ illlons d'utllssateurs à travers le monde en octubre 1994. Ces utllisateurs sont dles unhuersilialies, des employes gonvernementaux ou d'entreprises mals ansst le girand public. Ses tentacules ne cessent de s'étendre. (qu'on pense icl an secteur commercial qui tente de mener ces acturtés commerclales à l'Intérleur de ce réseau.

Pour le chercheilr, INTERNET offre dilférent.s services tels que le courrier électronlque. des forums spéclallsés. le télédéchargement de fichlers. et une inflntté de ressources dont notamment les catalogues des blbllothèques unlversltaires. C'est à un survol rapide de ces dlfférents services et. à quelyues lessources (jue nous convions malutenant ie Iecteil,

Le commler électromalue permet de commungater avec d'antives lulerloculent's à travers le incmide vla des postes de

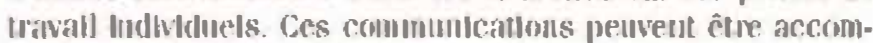

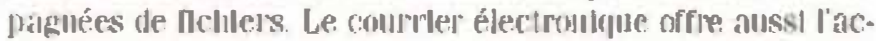
cès à des forums porlant sur différents sujets des plus sérleux aux plus excotlques. Toutes ces counmulcatlous seffectuent glatultement sl laccès que vous possédez se falt par l'Intermédialre d'une inst.tution unlversltalre. Voyons yuelques ressources fiomumentaires gul selon nous mérltent l'altentlon dı chers:lselan:

II est possible à parth d'UNCoVER de consulter des tables dles matlères de plus de 14.000 tutress de périodlloues reçus par les blbllothèues particlpant a ce consortlum. La Imajorllé de ces remies sont recensés depuls 1987 et. envirun 4 ,ono articles y sont aloutés quotldennement. De plus. UNCoN'ER offre le service de IIvalson d'articles de revues qul fonctloume cependinut. stul une base commerclale. (Pour y accécler l'adresse électrontque litml est: lutp:/Aww.blb.uc|am .c.RINCOVER.htunl).

Current Contents est une antre ressource pernothant. de consulter des tltues d'altikles de pérlodlipues recherchables par sujet. Ce service pour le moment semble décevolr car le inchle de repermge ne semble pas an polnt. (Je vous laisse

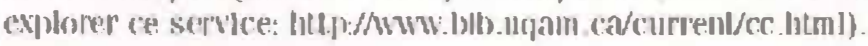

PIusleur's sltes sur liNTERNET présentent, fles listes de tures de documents par secteur: documents en économle par exemple, ell tourlsme, etc. D'antues sltes offrent le texte de documents: des journaux, des pérlodiluues. des documents prodults par différents gouvernements. etc. En falt. les ressources sur INTERNET soul. multules et aे cliacue four s'aloutent d'autres ressources. Slgnalons qu'une recherche par sulet est fort heurensement possible grâce à des outfls cle navlgatlon l.els que GoPHER (qui présente par menus difPéıents accès principalement. daus les unlversites) VERONICA Isel'blce de recheırlie à l'lutél'leur' des mentis présentés par GOPHER) et. le Would Whle Web appreté anssl wwW (service de lecherclie le plus populalre à l'beture actuelle sur INTERNETI. Lutllisat.Ion de l'un de ces trols services devralent vous permetlent. de juger par vous-me une de l'urtmensité de ce cyber espace de j'inforinatlon.

Notes

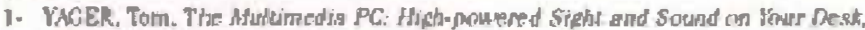
Bste. (Fels. 1952). pp.217-226.

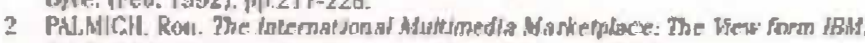

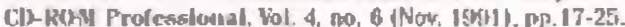

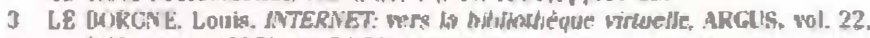

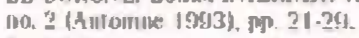

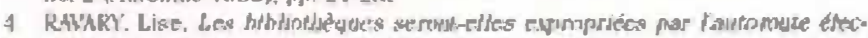

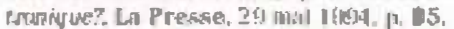

5 ComPER. W. S.. Gettiag Beyand Dooje, Informallon Proceaning

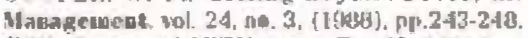

b JUHL. BeCh and LOWRY, Anifa. The CD-ROM Revolutans at Columblas year whe. Serlak Lbrarlan. vol. 17. No. 3/4 \{1900\}. np.69-80.

7 SCHULTZ, Kim and SALOMON, Kiristine. End lisers Reposd to CO-ROM,

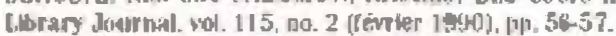

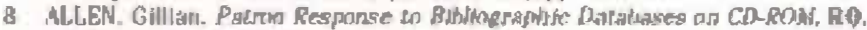
vol. 29. no. I (Fall 1909). pp. 103.110.

9 The CD-ROM Disecent, London: TPPL. ISSS.

10 CDRtoAss in f'rine. Wertport: Mechler. (Anumel) 1987-; Gale Directery of

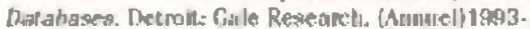

